\title{
Bidirectional electron conic observations for photoelectrons in the Martian ionosphere
}

\author{
YuTian Cao ${ }^{1,2}$, Jun Cui ${ }^{3,4}{ }^{*}$, BinBin $\mathrm{Ni}^{5,4}$, XiaoShu Wu, ${ }^{1,4}$, Qiong $\mathrm{Luo}^{5}$, and ZhaoGuo $\mathrm{He}^{3,4}$ \\ ${ }^{1}$ Key Laboratory of Lunar and Deep Space Exploration, National Astronomical Observatories, Chinese Academy of Sciences, Beijing 100012, China; \\ ${ }^{2}$ School of Astronomy and Space Sciences, University of Chinese Academy of Sciences, Beijing 100049, China; \\ ${ }^{3}$ School of Atmospheric Sciences, Sun Yat-sen University, Zhuhai, Guangdong 519082, China; \\ ${ }^{4}$ Center for Excellence in Comparative Planetology, Chinese Academy of Sciences, Hefei 230026, China; \\ ${ }^{5}$ Department of Space Physics, School of Electronic Information, Wuhan University, Wuhan 430072, China \\ Key Points: \\ - An analysis is conducted focusing on the bidirectional electron conics at 19-55 eV on Mars \\ - BECs are more likely observed on the nightside and near strong crustal magnetic anomalies \\ - 19-55 eV BECs are formed due to day-to-night transport along cross-terminator magnetic field lines
}

Citation: Cao, Y. T., Cui, J., Ni, B. B., Wu, X. S., Luo, Q., and He, Z. G. (2020). Bidirectional electron conic observations for photoelectrons in the Martian ionosphere. Earth Planet. Phys., 4(4), 403-407. http://doi.org/10.26464/epp2020037

\begin{abstract}
Electron pitch angle distributions similar to bidirectional electron conics (BECs) have been reported at Mars in previous studies based on analyses of Mars Global Surveyor measurements. BEC distribution, also termed "butterfly" distribution, presents a local minimum flux at $90^{\circ}$ and a maximum flux before reaching the local loss cone. Previous studies have focused on $115 \mathrm{eV}$ electrons that were produced mainly via solar wind electron impact ionization. Here using Solar Wind Electron Analyzer measurements made onboard the Mars Atmosphere and Volatile Evolution spacecraft, we identify 513 BEC events for 19-55 eV photoelectrons that were generated via photoionization only. Therefore, we are investigating electrons observed in regions well away from their source regions, to be distinguished from $115 \mathrm{eV}$ electrons observed and produced in the same regions. We investigate the spatial distribution of the 19-55 eV BECs, revealing that they are more likely observed on the nightside as well as near strong crustal magnetic anomalies. We propose that the 19-55 eV photoelectron BECs are formed due to day-to-night transport and the magnetic mirror effect of photoelectrons moving along cross-terminator closed magnetic field lines.
\end{abstract}

Keywords: Martian ionosphere; photoelectron; pitch angle distribution

\section{Introduction}

Unlike Earth, Mars does not have a global dynamo magnetic field; thus, the solar wind, carrying the interplanetary magnetic field (IMF), can interact with the Martian upper atmosphere directly and even reach the Martian ionosphere (Nagy et al., 2004). Suprathermal electrons in the ionosphere are typically magnetized with guiding center motions following the ambient magnetic field lines. The IMF that extends to the Martian ionosphere, therefore, affects the pitch angle distribution (PAD) of ambient suprathermal electrons. Pitch angle, which is the angle between the electron velocity and the magnetic field direction, indicates the direction of electron propagation along the magnetic field line. In practice, the guiding center motion and the PAD of suprathermal electrons in the vicinity of Mars is critically controlled by the ambient magnetic field configuration, which is characterized by either

Correspondence to: J. Cui, cuijun7@mail.sysu.edu.cn

Received 28 MAR 2020; Accepted 29 APR 2020.

Accepted article online 12 JUN 2020.

(C) 2020 by Earth and Planetary Physics. open field lines in regions with weak crustal magnetic fields or closed field lines near strong crustal magnetic anomalies (e.g. Xu SS et al., 2017a, b, 2019; Weber et al., 2017). Several types of suprathermal electron PAD were identified first by Brain et al. (2007) based on electron spectral data from the Electron Reflectometer (ER) onboard the Mars Global Surveyor (MGS) (Acuña et al., 1992; Mitchell et al., 2001). More recently, several studies have focused on the suprathermal electron PAD with the aid of energetic electron measurements made by the Solar Wind Electron Analyzer (SWEA) onboard the Mars Atmosphere and Volatile Evolution (MAVEN) spacecraft and have characterized the Martian magnetic topology (e.g. Weber et al., 2017; Xu SS et al., 2019).

Among the types of energetic electron PAD, the bidirectional electron conic (BEC) distribution or "butterfly" distribution presents a local minimum flux at $90^{\circ}$ and a maximum flux before reaching the local loss cone (Brain et al., 2007). Such an interesting PAD has been observed and well-studied in the radiation belts of the Earth (e.g. Lundin et al., 1987; Menietti and Weimer, 1998; Gu XD et al., 2011; Chen Y et al., 2014; Ni BB et al., 2016; Yang C et al., 2017) and Jupiter (e.g. Ma Q et al., 2017; Allegrini et al., 2017). 
BECs in the Martian atmosphere were studied by Ulusen et al. (2011) but only for the 115 eV electrons. Ulusen et al. (2011) reported that these butterfly-distributed electrons were observed mainly in darkness, and that upstream conditions, including the draped IMF direction, the solar wind pressure, and the solar Extreme Ultraviolet (EUV) flux, did not affect observation of the events.

Previous studies on energetic electron PAD in the Martian ionosphere usually have focused on electrons at energies above 100 $\mathrm{eV}$, which are produced via either photoionization or solar wind electron impact ionization (e.g. Brain et al., 2007; Ulusen et al., 2011; Xu SS et al., 2016; 2017a, b, 2019; Weber et al., 2017). In this work, we identify BECs for photoelectrons at lower energies, which are generated through photoionization only. The discrepancy between the original sources of electrons in these events may lead to different BEC characteristics and different formation mechanisms.

\section{Dataset and Methodology}

The identification of BECs is based on differential energetic electron intensity measurements made by the MAVEN SWEA instrument (Mitchell et al., 2016). The SWEA is a symmetric hemispheric electrostatic analyzer that measures the suprathermal electron intensity at different energies, covering the range of $3 \mathrm{eV}-4.6 \mathrm{keV}$ with a resolution of $17 \%(\Delta E / E)$. It can also measure the arrival direction of electrons within a field of view (FOV) of $360^{\circ} \times 120^{\circ}$ of which $8 \%$ is blocked by the spacecraft body. The photoelectron pitch angle is determined by combining SWEA data and magnetic field data measured by the MAVEN Magnetometer (MAG) (Connerney et al., 2015). The data used here include the SWEA level 2 differential electron intensities accumulated from 18 October 2014 to 14 December 2017, spanning over one and a half Martian years.

To identify BEC photoelectrons, we first determine the integrated number fluxes of electrons over the energy range of 19-55 eV. The integrated fluxes at different pitch angles are calculated separately and are normalized by the averaged integrated flux. BEC events are identified based on these normalized fluxes with the following criteria: If the maximum normalized flux at moderate pitch angle (between $36^{\circ}$ and $72^{\circ}$ and between $108^{\circ}$ and $144^{\circ}$ ) is at least $2 \sigma$ (two standard deviations) greater than the minimum parallel flux (at pitch angle $<36^{\circ}$ ) and greater than the minimum antiparallel flux (at pitch angle $>144^{\circ}$ ), as well as greater than the minimum perpendicular flux (pitch angle between $72^{\circ}$ and $108^{\circ}$ ), the observed SWEA spectrum is classified as a BEC. An additional requirement for this category is that the minimum fluxes at moderate pitch angles defined above should be greater than the maximum fluxes of parallel, antiparallel, and perpendicular electrons. Our experience with SWEA data manipulation has found that this requirement is necessary for excluding undesired cases with fluctuating fluxes at moderate pitch angles.

We apply the above method to the entire SWEA dataset below $700 \mathrm{~km}$, the location of the averaged Martian photoelectron boundary (e.g. Garnier et al., 2017; Han QQ et al., 2019), which leads to the identification of 513 cases of BEC observations, on both the dayside and nightside of Mars. One typical example of a BEC detected during MAVEN orbit \#4494 at 04: 45: 34 UT (universal time) on 22 January 2017 is shown in Figure 1. The top panel shows the normalized electron flux integrated over the energy range of $19-55 \mathrm{eV}$ as a function of electron pitch angle, showing that clear enhancements at oblique angles peaked at $60^{\circ}$ and $130^{\circ}$. The displayed event occurred at $420 \mathrm{~km}$ with a solar zenith angle (SZA) of $137^{\circ}$ in the deep nightside Martian ionosphere. It is well known that photoelectrons produced on the dayside are characterized by discrete energy peaks near 22 and $27 \mathrm{eV}$ due to the strong solar Hell emission feature at $30.4 \mathrm{~nm}$ (Frahm et al., 2006, Wu XS et al., 2019a). The bottom panel presents the differential energy flux of electrons with a pitch angle of $59^{\circ}$, displaying such distinctive photoelectron energy peaks. This observation of photoelectrons in the deep nightside is indicative of day-to-night transport (Xu SS et al., 2016, Cao YT et al., 2020). Indeed, it is well known that day-to-night transport is an important source of plasma in the nightside Martian ionosphere, especially near the terminator (e.g. Zhang et al., 1990; Withers et al., 2012), and that such a process occurs mainly within a time duration of $5000 \mathrm{~s}$ after terminator crossing (Cui J et al., 2015) causes a clear dawn-dusk asymmetry in ion distribution (Girazian et al. 2017), and appears to be substantially suppressed by the presence of strong crustal magnetic anomalies (Cao YT et al., 2019). This study further demonstrates that day-to-night transport also affects the PAD of suprathermal electrons.
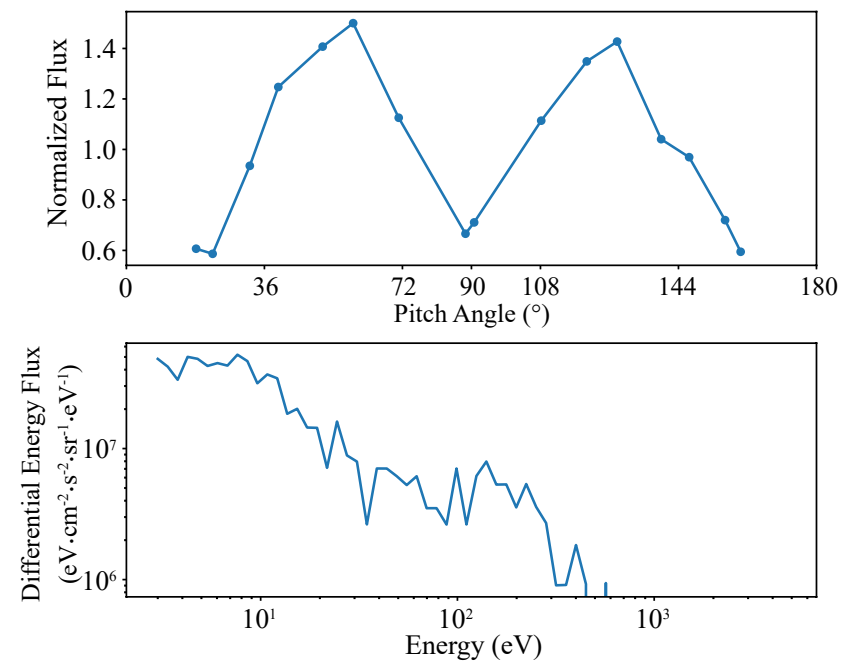

Figure 1. An example of an identified BEC pitch angle distribution of electrons over the energy range of 19-55 eV, measured at 04:45:34 UT (universal time) on 22 January 2017. Top: the normalized PAD of measured electrons; bottom: the differential energy flux of electrons with a pitch angle of $59^{\circ}$.

\section{Observations of BEC Photoelectrons}

We present in Figure 2 the occurrence rate of BECs with respect to altitude over the range of $100-700 \mathrm{~km}$ with a binsize of $100 \mathrm{~km}$, and also with respect to SZA over the range of $0^{\circ}-180^{\circ}$ with a binsize of $20^{\circ}$. The occurrence rate is defined as the ratio of $\mathrm{BEC}$ observations to the total number of electron measurements within the full $180^{\circ}$ pitch angle coverage. For photoelectrons over the 
energy range of 19-55 eV, BECs are rarely observed below $300 \mathrm{~km}$, as the occurrence rate of BEC measurements is less than $0.4 \%$ at all SZA bins on both the dayside and nightside. At altitudes above $300 \mathrm{~km}, \mathrm{BEC}$ events are more easily found over the SZA range of $80^{\circ}-140^{\circ}$. In particular, the occurrence rate of BECs identified in the bin with SZA from $120^{\circ}$ to $140^{\circ}$ and with altitude from $500 \mathrm{~km}$ to $600 \mathrm{~km}$ is over $1.2 \%$. On the dayside with SZA less than $60^{\circ}, \mathrm{BEC}$ event occurrence rates are less than $0.2 \%$ at all altitudes.

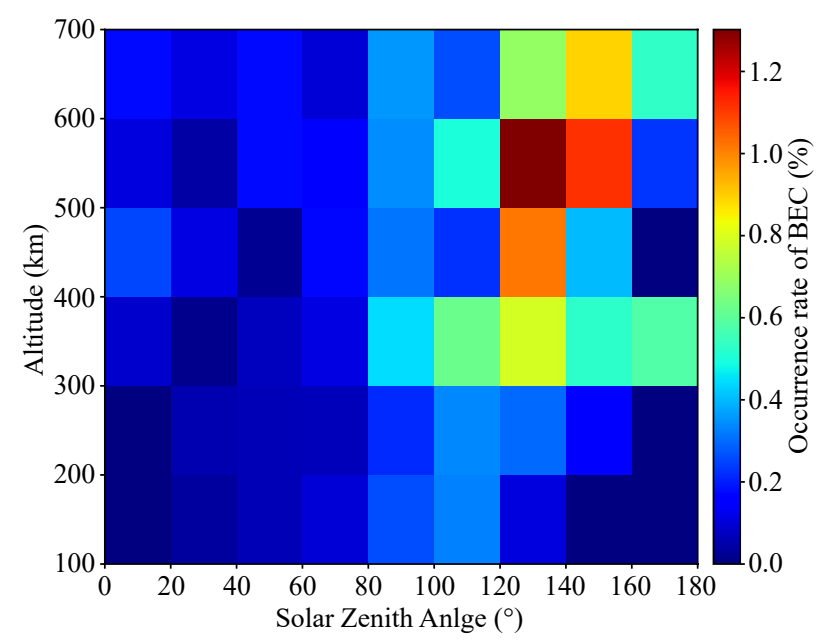

Figure 2. The occurrence rate of BEC observations as a function of altitude over the range of $100-700 \mathrm{~km}$ with a binsize of $100 \mathrm{~km}$, and as a function of SZA over the range of $0^{\circ}-180^{\circ}$ with a binsize of $20^{\circ}$. The occurrence rate is defined as the ratio of $\mathrm{BEC}$ observations to the total number of electron measurements within the full $180^{\circ}$ pitch angle coverage.

Because photoelectrons are originally produced by photoionization, isotropic PADs for these primary photoelectrons are expected. The photoelectron exobase is located at $\sim 160 \mathrm{~km}$ (Xu SS et al., 2016), below which sufficient collisions result in an isotropic PAD. Above the exobase, transport dominates over local production and energy degradation (Wu XS et al., 2019b). The low occurrence of $B E C$ photoelectrons on the dayside indicates that regional photoelectron transport purely on the dayside is not responsible for the majority of BEC observations reported in this study.

Most of the BEC photoelectrons are found with SZA above $80^{\circ}$, where the effect of photoionization is diminished and day-tonight transport becomes dominant near the terminator and on the nightside (Zhang et al., 1990; Fox et al., 1993; Němec et al., 2010; Duru et al., 2011; Withers et al., 2012; Cui J et al., 2015; Cao YT et al., 2019). Unlike the other processes, day-to-night transport is complicated; it may be driven by the thermospheric wind field at low altitudes (Bougher et al., 2015; Adams et al., 2018) and by the plasma pressure gradient at high altitudes (Ma YJ et al., 2014). Besides, Wu XS et al. (2019c) argued that the momentum budget of the ionospheric plasma on Mars is likely to be dominated by the magnetic pressure gradient (see also Hamil et al. 2019).

Here we propose that the preferred observations of BECs are related to day-to-night photoelectron transport across the terminator. Since such a transport is critically controlled by the ambient magnetic field topology (Cao YT et al., 2019), we present the geographic maps of the BEC measurements to visualize the effect of the planet-fixed crustal magnetic fields in Figure 3. Figure 3 shows the statistics of BEC occurrence rate as a function of longitude and latitude in $30^{\circ} \times 30^{\circ}$ bins; the crustal magnetic field model of Morschhauser et al. (2014), appropriate for the altitude of $400 \mathrm{~km}$, is superimposed for reference.

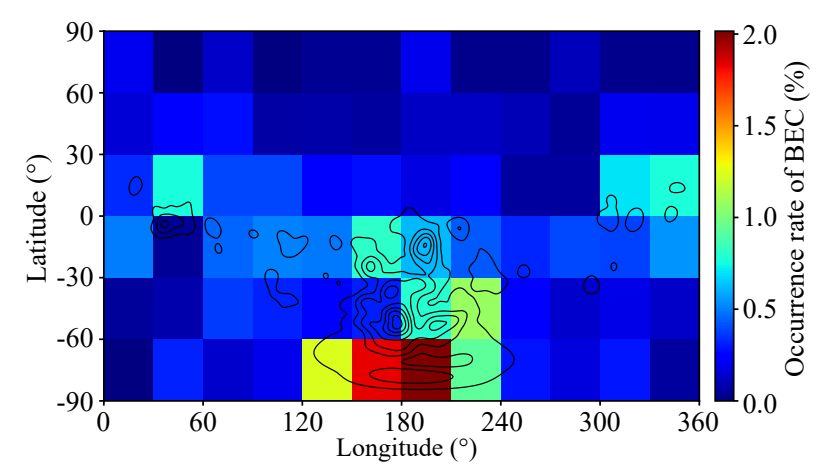

Figure 3. The occurrence rate of $\mathrm{BEC}$ photoelectrons as a function of longitude and latitude in $30^{\circ} \times 30^{\circ}$ bins. The crustal magnetic field model of Morschhauser et al. (2014), appropriate for the altitude of $400 \mathrm{~km}$, is superimposed for comparison.

Generally speaking, BECs are more easily observed over regions with strong crustal magnetic fields. For instance, the BEC occurrence rate is greater than $0.5 \%$ in each bin near strong magnetic fields. For regions located far away from crustal magnetic anomalies, such as the northern high latitude regions, BECs are less likely to be observed, with an averaged occurrence rate of $0.2 \%$. The approximate correlation between the distributions of BECs and crustal magnetic fields further supports our conjecture that the occurrence of photoelectron BECs is magnetically controlled. The region at latitudes between $-30^{\circ}$ and $-60^{\circ}$ and at longitudes between $120^{\circ}$ and $180^{\circ}$ appears to be an exception, with very few BEC observations. We expect such an anomaly to be caused by small number statistics, but this needs to be verified in the future when more SWEA data for this region have been accumulated.

\section{Discussion and Concluding Remarks}

By mapping the spatial distribution of measured BEC events, we find that most of the BECs are observed on the nightside as well as within regions characterized by strong crustal magnetic anomalies. These conclusions are consistent with the statistics obtained by Ulusen et al. (2011) based on the MGS ER measurements of 115 eV electrons. Ulusen et al. (2011) proposed that the time scales of the trapping and drift of $115 \mathrm{eV}$ electrons were much shorter than the planet's rotation period, indicating that the observed BECs were generated on the nightside shortly before being detected. They also proposed two possible mechanisms to explain the observed BECs: (1) the merging of open field lines neighboring the closed field lines; (2) the diffusing and/or scattering of electrons from open field lines into closed field lines. Their analyses were based on measurements of energetic electrons, which are more likely produced by solar wind electron precipitation on the nightside. However, in our study, we focus on electrons over a lower 
energy range, 19-55 eV, which are more likely produced by solar EUV ionization on the dayside. The above comparison indicates that the BECs analyzed by Ulusen et al. (2011) were observed in exactly the same regions where they were produced, whereas, in our case, the BECs are observed in regions well away from their source regions.

We manually check the electron energy spectra of all BECs identified on the nightside of Mars and find that more than $40 \%$ show clear photoelectron energy peaks in one or more angular spectra. These electrons with clear photoelectron energy peak signatures cannot be generated on the nightside but must be produced on the dayside by photoionization and then transported to the nightside. When photoelectrons are generated by solar EUV radiation at the feet of closed field lines, these photoelectrons bounce between the two feet of the field lines because of conservation of the first adiabatic invariant and the magnetic mirror effect. During such a process, photoelectrons with pitch angles close to $0^{\circ}$ or $180^{\circ}$ are able to enter the deep atmosphere and are lost quickly due to their collisions with ambient neutrals (Wu XS et al., 2019b). Electrons with pitch angles close to $90^{\circ}$ tend to stay near the minimum magnetic field point and cannot go far away before mirroring. We thus expect to see BECs along the field line at all positions between each of the two feet and the minimum magnetic field point. Such a scenario is more prominent on the nightside due to the lack of local production.

With the aid of angular differential energetic electron intensity measurements made by the SWEA onboard MAVEN, we identified $513 \mathrm{BEC}$ events for electrons over the energy range of 19-55 eV. We present the spatial distribution of these BECs with respect to altitude, SZA, and geographic latitude and longitude, revealing that BECs are more likely to be observed on the nightside and near strong crustal magnetic anomalies. These findings are consistent with the statistic survey of more energetic electrons at 115 eV made by Ulusen et al. (2011). However, due to different production sources of electrons at different energies, we propose that the formation of $19-55$ ev BEC photoelectrons is due to dayto-night transport and the magnetic mirror effect of electrons along cross terminator closed magnetic field lines, to be distinguished from the scenario proposed by Ulusen et al., (2011). Our analysis also illustrates that the measured photoelectron PADs should be different at different locations along their routes of dayto-night transport.

\section{Acknowledgments}

This work is supported by the B-type Strategic Priority Program No. XDB4100000 funded by the Chinese Academy of Sciences and the pre-research projects on Civil Aerospace Technologies No. D020105 and D020104 funded by China's National Space Administration. The authors also acknowledge support from the National Natural Science Foundation of China through grants 41525015, 41774186, and 41904154. The SWEA data used in the present study are publicly available at the MAVEN Science Data Center (https://lasp.colorado.edu/maven/sdc/public/).

\section{References}

Acuña, M. H., Connerney, J. E. P., Wasilewski, P., Lin, R. P., Anderson, K. A.,
Carlson, C. W., McFadden, J., Curtis, D. W., Réme, H., ... Bauer, S. J. (1992). Mars observer magnetic fields investigation. J. Geophys. Res, 97(E5), 7799-7814. https://doi.org/10.1029/92JE00344

Adams, D., Xu, S., Mitchell, D. L., Lillis, R. L., Fillingim, M., Andersson, L., Fowler, C., Connerney, J. E. P., Espley, J., and Mazelle, C. (2018). Using magnetic topology to probe the sources of Mars' nightside ionosphere. Geophys. Res. Lett., 45(22), 12,190-12,197. https://doi.org/10.1029/2018GL080629

Allegrini, F., Bagenal, F., Bolton, S., Connerney, J., Clark, G., Ebert, R. W., Kim, T. K., Kurth, W. S., Levin, S., ... Zink, J. L. (2017). Electron beams and loss cones in the auroral regions of Jupiter. Geophys. Res. Lett., 44(14), 7131-7139. https://doi.org/10.1002/2017GL073180

Bougher, S. W., Pawlowski, D., Bell, J. M., Nelli, S., McDunn, T., Murphy, J. R., Chizek, M., and Ridley, A. (2015). Mars global ionosphere-thermosphere model: solar cycle, seasonal, and diurnal variations of the mars upper atmosphere. J. Geophys. Res.: Planets, 120(2), 311-342. https://doi.org/10.1002/2014JE004715

Brain, D. A., Lillis, R. J., Mitchell, D. L., Halekas, J. S., and Lin, R. P. (2007). Electron pitch angle distributions as indicators of magnetic field topology near Mars. J. Geophys. Res.: Space Phys., 112(A9), A09201. https://doi.org/10.1029/2007JA012435

Cao, Y. T., Cui, J., Wu, X. S., Guo, J. P., and Wei, Y. (2019). Structural variability of the Nightside Martian ionosphere near the terminator: implications on plasma sources. J. Geophys. Res.: Planets, 124(6), 1495-1511. https://doi.org/10.1029/2019JE005970

Cao, Y. T., Cui, J., Wu, X. S., and Zhong, J. H. (2020). Photoelectron pitch angle distribution near Mars and implications on cross terminator magnetic field connectivity. Earth Planet. Phys., 4(1), 17-22. https://doi.org/10.26464/epp2020008

Chen, Y., Friedel, R. H. W., Henderson, M. G., Claudepierre, S. G., Morley, S. K., and Spence, H. E. (2014). REPAD: An empirical model of pitch angle distributions for energetic electrons in the Earth's outer radiation belt. J. Geophys. Res.: Space Phys., 119(3), 1693-1708. https://doi.org/10.1002/2013JA019431

Connerney, J. E. P., Espley, J., Lawton, P., Murphy, S., Odom, J., Oliversen, R., and Sheppard, D. (2015). The MAVEN magnetic field investigation. Space Sci. Rev., 195(1-4), 257-291. https://doi.org/10.1007/s11214-015-0169-4

Cui, J., Galand, M., Yelle, R. V., Wei, Y., and Zhang, S. J. (2015). Day-to- night transport in the Martian ionosphere: Implications from total electron content measurements. J. Geophys. Res.: Space Phys., 120(3), 2333-2346. https://doi.org/10.1002/2014JA020788

Duru, F., Gurnett, D. A., Morgan, D. D., Winningham, J. D., Frahm, R. A., and Nagy, A. F. (2011). Nightside ionosphere of Mars studied with local electron densities: A general overview and electron density depressions. J. Geophys. Res.: Space Phys., 116(A10), A10316. https://doi.org/10.1029/2011JA016835

Fox, J. L., Brannon, J. F., and Porter, H. S. (1993). Upper limits to the nightside ionosphere of Mars. Geophys. Res. Lett., 20(13), 1339-1342. https://doi.org/10.1029/93GL01349

Frahm, R. A., Winningham, J. D., Sharber, J. R., Scherrer, J. R., Jeffers, S. J., Coates, A. J., Linder, D. R., Kataria, D. O., Lundin, R., ... Dierker, C. (2006). Carbon dioxide photoelectron energy peaks at Mars. Icarus, 182(2), 371-382. https://doi.org/10.1016/j.icarus.2006.01.014

Garnier, P., Steckiewicz, M., Mazelle, C., Xu, S., Mitchell, D., Holmberg, M. K. G., Halekas, J. S., Andersson, L., Brain, D. A., ... Jakosky, B. M. (2017). The Martian photoelectron boundary as seen by MAVEN. J. Geophys. Res.: Space Phys., 122(10), 10,472-10,485. https://doi.org/10.1002/2017JA024497

Girazian, Z., Mahaffy, P. R., Lillis, R. J., Benna, M., Elrod, M., and Jakosky, B. M. (2017). Nightside ionosphere of Mars: Composition, vertical structure, and variability. J. Geophys. Res.: Space Phys., 122(4), 4712-4725. https://doi.org/10.1002/2016JA023508

Gu, X. D., Zhao, Z. Y., Ni, B. B., Shprits, Y., and Zhou, C. (2011). Statistical analysis of pitch angle distribution of radiation belt energetic electrons near the geostationary orbit: CRRES observations. J. Geophys. Res.: Space Phys., 116(A1), A01208. https://doi.org/10.1029/2010JA016052

Hamil, O., Cravens, T. E., Rahmati, A., Connerney, J. E. P., and Andersson, L. (2019). Pressure gradients driving ion transport in the topside Martian atmosphere. J. Geophys. Res.: Space Phys., 124(7), 6117-6126. 
https://doi.org/10.1029/2019ja026670

Han, Q. Q., Fan, K., Cui, J., Wei, Y., Fraenz, M., Dubinin, E., Chai, L. H., Rong, Z. J., Wan, W. X., ... Connerney, J. E. P. (2019). The relationship between photoelectron boundary and steep electron density gradient on Mars: MAVEN observations. J. Geophys. Res.: Space Phys., 124(10), 8015-8022. https://doi.org/10.1029/2019JA026739

Lundin, R., Eliasson, L., Hultqvist, B., and Stasiewicz, K. (1987). Plasma ergization on auroral field lines as observed by the Viking spacecraft. Geophys. Res. Lett., 14(4), 443-446. https://doi.org/10.1029/GL014i004p00443

Ma, Y. J., Fang, X., Nagy, A. F., Russell, C. T., and Toth, G. (2014). Martian ionospheric responses to dynamic pressure enhancements in the solar wind. J. Geophys. Res. Space Physics, 119(2), 1272-1286. https://doi.org/10.1002/2013JA019402

Ma, Q., Thorne, R. M., Li, W., Zhang, X. J., Mauk, B. H., Paranicas, C., Haggerty, D. K., Kurth, W. S., Connerney, J. E. P., ... Bolton, S. J. (2017). Electron butterfly distributions at particular magnetic latitudes observed during Juno's perijove pass. Geophys. Res. Lett., 44(10), 4489-4496. https://doi.org/10.1002/2017GL072983

Menietti, J. D., and Weimer, D. R. (1998). DE observations of electric field oscillations associated with an electron conic. J. Geophys. Res.: Space Phys., 103(A1), 431-438. https://doi.org/10.1029/97JA02496

Mitchell, D. L., Lin, R. P., Mazelle, C., Rème, H., Cloutier, P. A., Connerney, J. E. P., Acuña, M.H., and Ness, N. F. (2001). Probing Mars' crustal magnetic field and ionosphere with the MGS Electron Reflectometer. J. Geophys. Res.: Planets, 106(E10), 23,419-23,427. https://doi.org/10.1029/2000JE001435

Mitchell, D. L., Mazelle, C., Sauvaud, J. A., Thocaven, J. J., Rouzaud, J., Fedorov, A., Rouger, P., Toublanc, D., Taylor, E., ... Jakosky, B. M. (2016). The MAVEN solar wind electron analyzer. Space Sci. Rev., 200(1-4), 495-528. https://doi.org/10.1007/s11214-015-0232-1

Morschhauser, A., Lesur, V., and Grott, M. (2014). A spherical harmonic model of the lithospheric magnetic field of Mars. J. Geophys. Res.: Planets, 119(6), 1162-1188. https://doi.org/10.1002/2013JE004555

Nagy, A. F., Winterhalter, D., Sauer, K., Cravens, T. E., Brecht, S., Mazelle, C., Crider, D., Kallio, E., Zakharov, A., ...Trotignon, J. G. (2004). The plasma environment of Mars. Space Sci. Rev., 111(1-2), 33-114. https://doi.org/10.1023/B:SPAC.0000032718.47512.92

Němec, F., Morgan, D. D., Gurnett, D. A., and Duru, F. (2010). Nightside ionosphere of Mars: Radar soundings by the Mars Express spacecraft. J. Geophys. Res.: Planets, 115(E12), E12009. https://doi.org/10.1029/2010JE003663

Ni, B. B., Zou, Z. Y., Li, X. L., Bortnik, J., Xie, L., and Gu, X. D. (2016). Occurrence characteristics of outer zone relativistic electron butterfly distribution: a survey of Van Allen Probes REPT measurements. Geophys. Res. Lett, 43(11), 5644-5652. https://doi.org/10.1002/2016GL069350

Ulusen, D., Brain, D. A., and Mitchell, D. L. (2011). Observation of conical electron distributions over Martian crustal magnetic fields. J. Geophys. Res.: Space Phys., 116(A7), A07214. https://doi.org/10.1029/2010JA016217
Weber, T., Brain, D., Mitchell, D., Xu, S. S., Connerney, J., and Halekas, J. (2017). Characterization of low-altitude Nightside Martian magnetic topology using electron pitch angle distributions. J. Geophys. Res.: Space Phys., 122(10), 9777-9789. https://doi.org/10.1002/2017JA024491

Withers, P., Fillingim, M. O., Lillis, R. J., Häusler, B., Hinson, D. P., Tyler, G. L., Pätzold, M., Peter, K., Tellmann, S., and Witasse, O. (2012). Observations of the nightside ionosphere of Mars by the Mars Express Radio Science Experiment (MaRS). J. Geophys. Res.: Space Phys., 117(A12), A12307. https://doi.org/10.1029/2012JA018185

Wu, X. S., Cui, J., Cao, Y. T., Liu, L. J., Zhou, Z. J., Huang, Y. Y., He, F., and Wei, Y. (2019a). On the hardness of the photoelectron energy spectrum near Mars. J. Geophys. Res.: Planets, 124(11), 2745-2753. https://doi.org/10.1029/2019JE006093

Wu, X. S., Cui, J., Yu, J., Liu, L. J., and Zhou, Z. J. (2019b). Photoelectron balance in the dayside Martian upper atmosphere. Earth Planet. Phys., 3(5), 373-379. https://doi.org/10.26464/epp2019038

Wu, X. S., Cui, J., Xu, S. S., Lillis, R. J., Yelle, R. V., Edberg, N. J. T., Vigren, E., Rong, Z. J., Fan, K., ... Mitchell, D. L. (2019c). The morphology of the topside Martian ionosphere: implications on bulk ion flow. J. Geophys. Res.: Planets, 124(3), 734-751. https://doi.org/10.1029/2018JE005895

Xu, S. S., Mitchell, D., Liemohn, M., Dong, C. F., Bougher, S., Fillingim, M., Lillis, R., McFadden, J., Mazelle, C., ... Jakosky, B. (2016). Deep nightside photoelectron observations by MAVEN SWEA: Implications for Martian northern hemispheric magnetic topology and nightside ionosphere source. Geophys. Res. Lett., 43(17), 8876-8884. https://doi.org/10.1002/2016GL070527

Xu, S. S., Mitchell, D., Liemohn, M., Fang, X. H., Ma, Y. J., Luhmann, J., Brain, D., Steckiewicz, M., Mazelle, C., ... Jakosky, B. (2017). Martian low-altitude magnetic topology deduced from MAVEN/SWEA observations. J. Geophys. Res.: Space Phys., 122(2), 1831-1852. https://doi.org/10.1002/2016JA023467

Xu, S. S., Mitchell, D., Luhmann, J., Ma, Y. J., Fang, X. H., Harada, Y., Hara, T., Brain, D., Weber, T., ... DiBraccio, G. A. (2017). High-altitude closed magnetic loops at mars observed by MAVEN. Geophys. Res. Lett., 44(22), 11,229-11,238. https://doi.org/10.1002/2017GL075831

Xu, S. S., Weber, T., Mitchell, D. L., Brain, D. A., Mazelle, C., DiBraccio, G. A., and Espley, J. (2019). A technique to infer magnetic topology at mars and its application to the terminator region. J. Geophys. Res.: Space Phys., 124(3), 1823-1842. https://doi.org/10.1029/2018JA026366

Yang, C., Su, Z. P., Xiao, F. L., Zheng, H. N., Wang, Y. M., Wang, S., Spence, H. E., Reeves, G. D., Baker, D. N.,... Funsten, H. O. (2017). A positive correlation between energetic electron butterfly distributions and magnetosonic waves in the radiation belt slot region. Geophys. Res. Lett., 44(9), 3980-3990. https://doi.org/10.1002/2017GL073116

Zhang, M. H. G., Luhmann, J. G., and Kliore, A. J. (1990). An observational study of the nightside ionospheres of Mars and Venus with radio occultation methods. J. Geophys. Res.: Space Phys., 95(A10), 17,095-17,102. https://doi.org/10.1029/JA095iA10p17095 